\title{
Toxoplasmic Encephalitis in an HIV Infected Pregnant Woman: Successful Outcome for Both Mother and Child
}

\author{
Susie Andries Nogueira, Ana Lúcia Guedes, \\ Elizabeth S. Machado, Juliana A. Matos, \\ Tomás P. Costa, Ely M. Cortes and John S. Lambert
}

\author{
Clementino Fraga Filho Hospital, Federal University \\ of Rio de Janeiro; Hospital of Lagoa, Rio de Janeiro, \\ RJ, Brazil; Institute of Human Virology, Univ. of \\ Maryland, USA
}

\begin{abstract}
This report describes a case of Toxoplasma encephalitis during pregnancy of an HIV infected woman who was severely immunosuppressed $\left(\mathrm{CD}_{4}: 17 \mathrm{cells} / \mathrm{mm} 3\right)$, had a high viral load (RNA PCR:230,000 copies/ $\mathrm{ml}$ ), was treated with sulfadiazine, pyrimethamine and folinic acid for toxoplasmosis and was being treated with highly potent antiretroviral drugs (AZT, 3TC and nelfinavir) for HIV infection. The newborn was born through an elective $\mathrm{C}$-section, received six weeks of AZT according to the 076 protocol and was clinically normal at birth. Subsequently he had two RNA PCR negatives for HIV, seroreverted and had no clinical or laboratory evidence of congenital toxoplasmosis. Despite the concerns of the use of these combined therapies on the foetus during pregnancy, their efficacy illustrates that keeping the mother alive and in good health is an important strategy to protect the unborn child from acquiring these two infections. Key Words: Toxoplasma encephalitis, pregnancy, HIV/AIDS, prevention of vertical transmission.
\end{abstract}

\section{Case Report}

KRCG, 24 years old, female, received the diagnosis of HIV infection in November 1999; and in January 2000 was admitted to HL, Rio de Janeiro, Brazil with a diagnosis of Pneumocystis carinii pneumonia (PCP) and was found to be pregnant. Following treatment of her PCP, she was discharged on HAART (AZT/3TC and nelfinavir) and was provided with prophylactic treatment for PCP(SMZ/TMP), to which she had poor adherence. In February 2000 she was admitted to the HUCFF, Inf. Diseases Service, with a history of six days of headache, nausea, vomiting, and 48 hours prior to admission she had fever, a decrease in

Received on 15 December 2001; revised 18 June 2002.

Address for correspondence: Dr. Susie Andries Nogueira. Serviço de Doenças Infecciosas e Parasitárias - $5^{\circ}$ andar HUCFF/UFRJ. Avenida Brigadeiro Trompowski s/n - Ilha do Fundão.Rio de Janeiro- CEP: 21941.590 - RJ . Brasil. E-mail: susie@hucff.ufrj.br Fax:(55 21)2590-5422.

The Brazilian Journal of Infectious Diseases 2002;6(4):201-205 (C) 2002 by The Brazilian Journal of Infectious Diseases and Contexto Publishing. All rights reserved. $1413-8670$ consciousness, but no history of seizures. On physical examination she was stuporous, had diminished strength on her left side, was anaemic, dehydrated, but without respiratory or circulatory abnormalities. BP: 110x60 mm Hg; pulse: $72 / \mathrm{min}$; resp. rate: $26 / \mathrm{min}$; axillary temperature: $37.4^{\circ} \mathrm{C}$; had evidence of oral candidiasis and pruritus of the lower limbs, and was 29-30 weeks pregnant. Laboratory results at admission: urea: 25.9; glucose: 125; TGO: 19, amylase; 72, leukocytes: 5800 with 63\% segs, $23 \%$ lymphs; platelets: 330,000 ; HG: 10.3; $\mathrm{CD}_{4}: 17$ cells $/ \mathrm{mm}^{3}(1 \%)$ and $\mathrm{CD}_{8}: 1038(63 \%)$; HIV RNA PCR viral load: 230,000 copies/ml (5.4 log). Serology: hepatitis C (-), HBsAg (-), anti-HBc (+); CMV: IgG (+), IgM(-); Rubella : Ig M (-), IgG $(+)$; VDRL (-); Toxoplasmosis: $\operatorname{IgM}(-)$; $\operatorname{IgG}(+)$; latex for Cryptococcus antigen in serum: negative.

CT scan (Figure 1): a mass lesion with annular contrast enhancement in the right hemisphere, with significant surrounding oedema and a mid-line shift. Right ethmoidal and sphenoidal sinusitis.

Abdominal ultrassonography (US) in January, 2000: no abnormality, 19-20 weeks of pregnancy. US in March, 2000: no abnormality, 34-35 weeks of gestation. 
Past history of syphilis in 1998 with VDRL: 1/32, treated with penicillin. VDRL in February, May and June, 2000: negative.

Clinical Course: with the results of the head CT scan a presumptive diagnosis of toxoplasma encephalitis (TE) was made and sulfadiazine (SD) $100 \mathrm{mg} / \mathrm{kg}$, with pyrimethamine(PMT) $100 \mathrm{mg}$ loading dose, was given for 3 days, then decreased to PMT $50 \mathrm{mg} /$ day, and folinic acid, $15 \mathrm{mg} /$ day. In addition she was treated with fluconazole for oral candidiasis and cefuroxime IV for sinusitis; HAART was initiated on the $2^{\text {nd }}$ day of hospitalisation. Mannitol and dexametazone were added on the following day due to clinical deterioration and at day two she developed coma and left hemiplegia, in association with dyspnea, agitation and the need for frequent aspiration of the upper airway. Blood gas: $\mathrm{PO}_{2}$ : 54.5; $\mathrm{PCO}_{2}: 23 ; \mathrm{O}_{2}$ Saturation: $91.6 \%$. There was an improvement in her neurological status by the $4^{\text {th }}$ day of toxoplasmosis treatment, fundoscopy was normal at that time, by the $6^{\text {th }}$ day of admission she was alert, and by the $8^{\text {th }}$ day of therapy dexametazone was discontinued. She was discharged in April, 2000, still receiving a full dose of SD,PMT and folinic acid to treat toxoplasmosis, and antiretroviral therapy consisting of AZT/3TC and nelfinavir. She continued to be seen at the prenatal clinic of the Federal University, which cares for HIV infected pregnant women, was continued on HAART and four weeks later was placed on toxoplasma maintenance therapy with SD: $2 \mathrm{~g} /$ day, PMT: $25 \mathrm{mg}$, and folinic acid every other day with good adherence and tolerance. In April, 2000, the patient's $\mathrm{CD}_{4}$ was: 35 cells (4\%); $\mathrm{CD}_{8}: 625$ (72\%) and RNA PCR for HIV: $<80$ copies (undetectable). An elective C-section was performed in June 2000 at 40 weeks of pregnancy, AZT intravenous was given during the C-section, and the baby received six weeks of oral AZT according to the 076 regimen. Mother was counselled not to breast-feed and baby formula was provided to the infant for 12 months. The baby's birth weight was $2,750 \mathrm{~g}$, height of $49 \mathrm{~cm}$, and head circumference of $34 \mathrm{~cm}$, APGARs were 8/9, and there were no abnormalities on physical examination suggestive of congenital toxoplasmosis, at which time spiramycin was presumptively started. Skull RX: normal; fundoscopic exam: normal; serology (ELISA) for toxoplasmosis was IgM (-) and Ig G (+): 178.9; positive HIV tests (ELISA and Western blot) were found. Spiramycin was subsequently discontinued after the diagnosis of congenital toxoplasmosis was ruled out. Neurological development was normal when seen at 15 month of age, a second fundoscopy at 15 months of age was negative, and the HIV RNA PCR was negative at 6 weeks and 3 months of age. ELISA for HIV was negative at 13 months of age. At most recent evaluation (November, 2001) both mother and child were under follow up, and mother was taking HAART and secondary prophylaxis for TE/pneumocystosis without evidence of disease reactivation, and the child had no evidence of congenital toxoplasmosis or HIV infection.

\section{Discussion}

Toxoplasma gondii is an ubiquitous obligatory widely-distributed intracellular protozoan. Once infected, the host normally acquires lifelong immunity induced by the persistence of the parasite in an encysted form. Toxoplasmosis in the immunocompetent person is usually of little consequence, and often asymptomatic. Reactivation of previous latent infection in an immunosuppressed host results in a varied clinical spectrum, predominantly affecting the nervous system [1]. A primary infection in a pregnant women has a risk of $40 \%$ of transmitting Toxoplasma gondii infection to the foetus. The risk is low in early pregnancy and only $6 \%$ (3\% to $9 \%$ ) at 13 weeks gestation. Thereafter the risk rises rapidly, reaching 40\% (34\% to $46 \%)$ at 26 weeks, $72 \%(61 \%$ to $80 \%)$ at 36 weeks and $81 \%$ just before delivery; major congenital abnormalities often occur in the first and second trimester [2,3].

However, in severely immunocompromised individuals, parasitemia occurs, as demonstrated by positive PCR in the blood of HIV patients infected with TE [4-6]; so it is possible that HIV-infected pregnant women with latent toxoplasmic infection, with negative IgM anti-toxoplasma antibodies, but with positive IgG, 
Figure 1. CT scan showing great mass lesion with edema

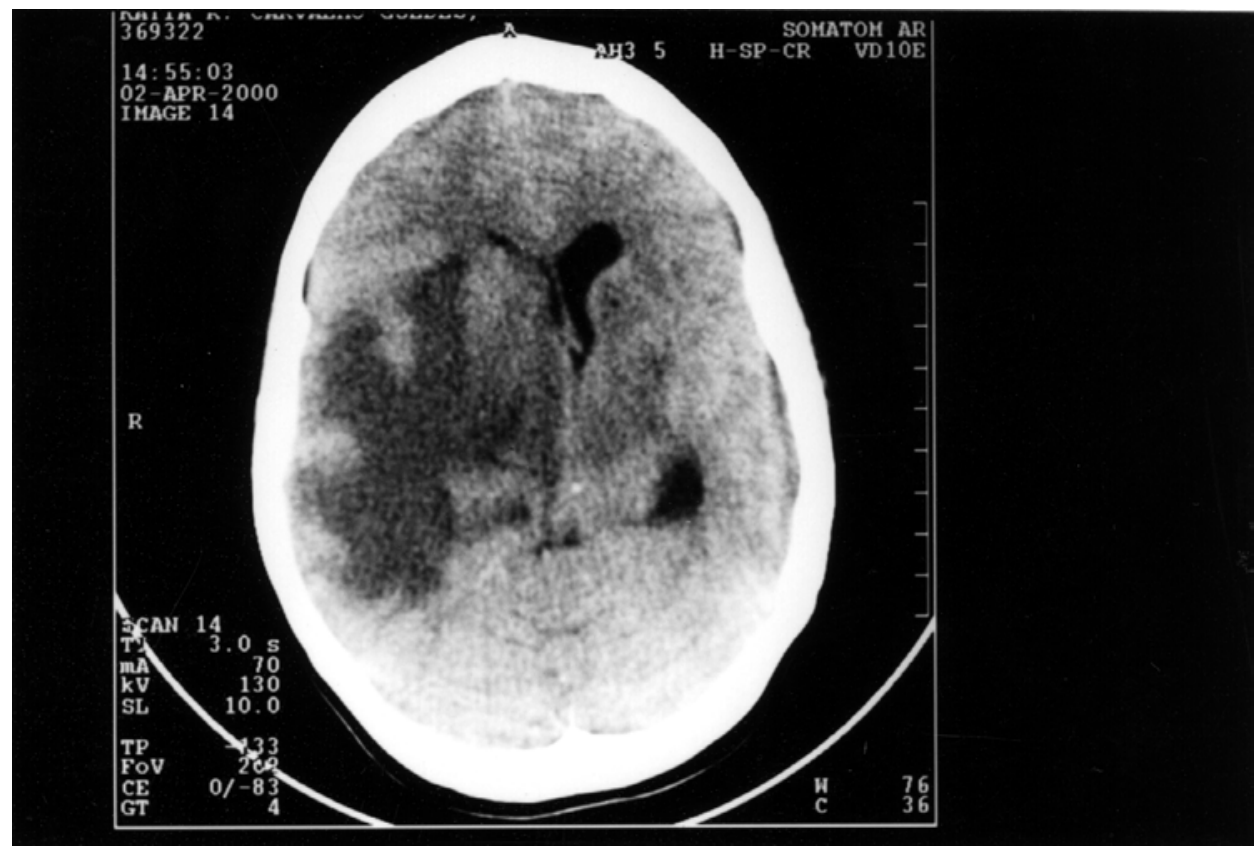

Figure 2. CT scan showing great mass lesion with annular caption after intravenous contrast in right hemisphere, with mid-line shift

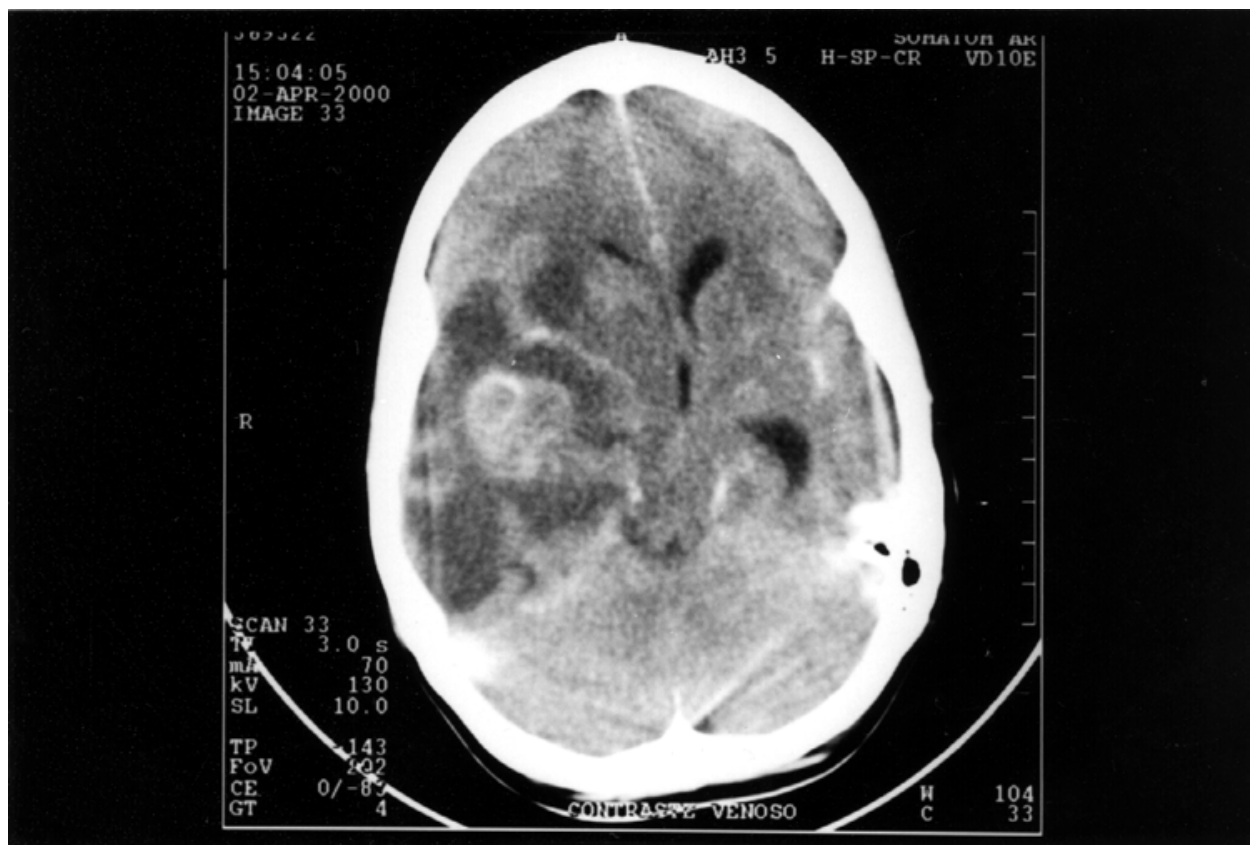


would transmit T. gondii to her child. The European Collaborative Network on Toxoplasmosis [7] published a note that there is a low incidence of congenital toxoplasmosis in children born from women infected with HIV. Among 1,058 children followed for a mean duration of 35 months, only one child developed clinical toxoplasmosis; she was also HIV infected. Congenital infection was excluded serologically in a subgroup of 167 children, of whom an estimated 71 had been at risk of infection. Probably transmission was low in this cohort because most of the HIV infected women who are pregnant are asymptomatic and have high levels of $\mathrm{CD}_{4}$, which makes the reactivation of a latent toxoplasmic infection very rare. However there are some reports of poor outcomes when an HIV infected woman has TE during pregnancy, with vertical transmission of one or both infections to the foetus and higher morbidity-mortality for the mother [8-11].

This case illustrates a severe case of toxoplasma encephalitis in an immunocompromised HIV infected pregnant woman who was at great risk for transmitting HIV (low $\mathrm{CD}_{4}$ and high viral load) [12] and Toxoplasma gondii infection to her child. Due to the foetal toxicicity of sulfonamide and pyrimethamine during gestation, as well as exposing the unborn child to radiation during CT scan, there was concern by the physicians who first examined this patient upon admission to HUCFF. With the presumptive diagnosis of TE, the health of the mother was prioritised, and specific therapy for toxoplasmosis was begun with standard doses [13]. Termination of pregnancy has been discussed in some European countries to prevent congenital toxoplasmosis, but such a measure is not allowed in our country, and the poor conditions of the patient did not permit any other intervention. Despite the initially poor clinical course, the patient recovered with toxoplasma therapy without sequels; and with antiretroviral combined therapy (HAART), the 076 regimen and elective $\mathrm{C}$ section, we were able to prevent the baby from contracting either infection. The prevalence of chronic infection by $T$. gondii [as measured by IgG-specific antibodies] in a cohort of $227 \mathrm{HIV}$ infected pregnant women-infant pairs followed in our Program is $71.4 \%$, and the mean $\mathrm{CD}_{4}$ is 428.4 cell/mm3, and no baby has been born with clinical congenital toxoplasmosis to date [14]. Additionally in those women with $\mathrm{CD}_{4}<200$ cells/mm3, cotrimoxazole is administered during pregnancy 3 times a week, and it is well demonstrated that this prophylactic regimen not only prevents pneumocystosis but also the reactivation of latent toxoplasmosis [13,15,16]; making congenital toxoplasmosis among our babies very rare.

This is the first reported case of simultaneous treatment of toxoplasma encephalitis in a woman with advanced immunosuppression and high viral load, with initiation of toxoplasma treatment and highly active antiretroviral therapy. There was low toxicity and excellent outcome for the pregnant mother and her child. Despite the concerns about the use of these combined therapies for the foetus during pregnancy, their efficacy illustrates that keeping the mother alive and in good health is an important strategy to protect the unborn child from acquiring these two infections. As both HIV and toxoplasmosis are more prevalent in developing world settings, it is mandatory, in these times of globalisation, that universal access to drugs for the poor patients be available, to allow for better outcomes for the mother and child when these and other infections are involved.

\section{References}

1. Remington J.S., McLeod R., Thulliez, Desmonts G. Toxoplasmosis. In: Remington J. et Klein J. [eds]. Infections Diseases of the fetus and newborn infant, Philadelphia: W.B. Saunders, 2000.

2. Gilbert R.E. Toxoplasmosis. In Newell M.L., McIntyre J., [eds]. Congenital and Perinatal Infections. Cambridge: Cambridge University Press, 2000.

3. Lebech M., Joynson D.H.M., Seitz H.M., et al. Classification system and case definition of $T$. gondii infection in immunocompetent pregnant women and their congenitally infected offspring. Eur J Clin Microbiol Infect Dis 1966; 15 (10):799-805.

4. Filice G., Hitt J., Mitchell C., et al. Diagnosis of Toxoplasma Parasitemia in Patients with AIDS by gene detection after amplification with polymerase Chain Reaction. J of Clin Microbiol 1993;31(9):2327-31.

5. Bierdermann K., Flepp M., Fiers W., et al. Pregnancy, immunosupression and rectivation of latent toxoplasmosis. J Perinat Med 1995;23:191-201. 
6. Dupouy-Camet J., Lavareda de Souza S., Maslo C., et al. Detection of Toxoplasma gondii in venous blood from AIDS patients by Polimerase Chain Reaction. J of Clin Microbiol 1993;31(7):1866-9.

7. European Collaborative Study and Research Network on congenital Toxoplasmosis: Low incidence of congenital toxoplasmosis in children born to women infected with human immunodeficiency virus. Eur J Obstetric Gynecol Reprod Biol 1996;68:93-6.

8. Mitchell C.D., Erlich S.S., Mastrucci M.T., et al. Congenital toxoplasmosis occuring in infants perinatally infected with human immunodeficiency virus. Pediatr Infect Dis J 1990;9:512.

9. Vanhems O.I., Hirschel B. Toxoplasmic encephalitis during pregnancy. AIDS 1993;7(1):142-3.

10. Marty P., Bongain A., Rahal A., et al.: Prenatal diagnosis of severe fetal toxoplasmosis as a result of of toxoplasma reactivation in an HIV-1 seropositive woman. Prenat Diagn 1994; 14:414-5.

11. O'Riordan, Farkas A.G. Maternal death due to cerebral toxoplasmosis British J of Obstetrics and Gynecology 1998; 105:565-6.

12. Mofenson L.M., Lambert J.S., Stiehm E.R., et al. Risk factors for perinatal transmission of HIV in women treated with ZDV. NEJM 1999;341:385-93.

13. McCabe R., Chirurgi V. Issues in toxoplasmosis. Inf Dis of North Am 1993;7(3):587-601.

14. Nogueira S. A., Machado E.S., Costa T.P., et al. Prevalência de co-infecções numa coorte de gestantes infectadas pelo HIV no Rio de Janeiro. Abstracts da IV Conferência Internacional sobre infecção pelo HIV em mulheres e crianças, 11e 12 de abril de 2002, Rio de Janeiro, p.28.

15. Derouin F., Jacqz-Aigrain E., Thulliez P., et al. Cotrimoxazole for prenatal treatment of congenital toxoplasmosis? Parasitology Today 2000;16(6): 254-6.

16. Bélanger F., et al. Incidence and risk factor of toxoplasmosis in a cohort of HIV infected patient-1980-1996. Clin Infect Dis 1999;28:575-81. 